\title{
OPERATORS SIMILAR TO THEIR ADJOINTS
}

JAMES P. WILLIAMS

This note is motivated by the following theorem of I. H. Sheth [4].

THEOREM. Let $T$ be a hyponormal operator $\left(T T^{*} \leqq T^{*} T\right)$ and suppose that $S^{-1} T S=T^{*}$ where $0 \notin W(S)^{-}$. Then $T$ is selfadjoint.

Here, and in what follows, all operators will be bounded linear transformations from a fixed Hilbert space into itself. $W(S)^{-}$is the closure of the numerical range $W(S)=\{\langle S x, x\rangle:\|x\|=1\}$ of $S$. We will make use of the fact that $W(S)^{-}$is convex and contains the spectrum $\sigma(S)$ of $S$.

The purpose of this note is to present a simple theorem which explains the above result. Before doing this, however, a few remarks are pertinent.

To begin with, the technique of [4] actually proves a much better result:

ThEOREM 1. If $T$ is any operator such that $S^{-1} T S=T^{*}$, where $0 \notin W(S)^{-}$, then the spectrum of $T$ is real.

Proof. It is clearly enough to show that the boundary of $\sigma(T)$ lies on the real axis. Since this is a subset of the approximate point spectrum of $T$, it suffices to show that if $\left(x_{n}\right)$ is a sequence of unit vectors such that $(T-\lambda) x_{n} \rightarrow 0$, then $\lambda$ is real. This latter assertion follows from the inequality

$$
\begin{aligned}
\left|(\bar{\lambda}-\lambda)\left\langle S^{-1} x_{n}, x_{n}\right\rangle\right| & =\left|\left\langle\left(T^{*}-\lambda\right) S^{-1} x_{n}, x_{n}\right\rangle-\left\langle\left(T^{*}-\bar{\lambda}\right) S^{-1} x_{n}, x_{n}\right\rangle\right| \\
& \leqq\left\|\left(T^{*}-\lambda\right) S^{-1} x_{n}\right\|+\left\|S^{-1}\right\|\left\|(T-\lambda) x_{n}\right\| \\
& =\left\|S^{-1}(T-\lambda) x_{n}\right\|+\left\|S^{-1}\right\|\left\|(T-\lambda) x_{n}\right\| \\
& \leqq 2\left\|S^{-1}\right\|\left\|(T-\lambda) x_{n}\right\|
\end{aligned}
$$

and the fact that $0 \notin W(S)^{-}$implies $0 \notin W\left(S^{-1}\right)^{-}$.

To recover Sheth's Theorem from Theorem 1 we need only observe that if $N$ is hyponormal, then $W(N)^{-}$is the convex hull of $\sigma(N)$.

It is worth noting that Theorem 1 includes a result of Beck and Putnam. To state this result, recall that a unitary operator $U$ is cramped [2] if $\sigma(U)$ is contained in an arc of the unit circle with central angle less than $\Pi$.

CoRollary [1], [2]. If $N$ is a normal operator which is unitarily

Received by the editors September 20, 1967. 
equivalent to its adjoint by a cramped unitary operator $U$, then $N$ is selfadjoint.

Proof. Since $U$ is normal, $W(U)^{-}$is the convex hull of the spectrum of $U$ and so the hypothesis insures that $0 \notin W(U)^{-}$.

Theorem 1 fails if the operator $S$ is merely required to be invertible. Even normality of both $S$ and $T$ does not help, as the following example shows:

Example 1. Let $T$ be the bilateral shift $\left(T e_{n}=e_{n+1}\right)$ on the span $l^{2}$ of the orthonormal set $\left\{e_{n}\right\}_{-\infty}^{+\infty}$ and let $S$ be the selfadjoint unitary defined by $S e_{n}=e_{-n}$. Then $S^{-1} T S=T^{-1}=T^{*}$, but the spectrum of $T$ is not real.

Here is the promised generalization:

Theorem 2. If $S^{-1} T S=T^{*}$ where $0 \notin W(S)^{-}$, then $T$ is similar to a selfadjoint operator.

Proof. Since $W(S)^{-}$is convex and does not contain 0 , we can separate 0 from $W(S)$ - by a half-plane. If necessary, we may replace $S$ by $e^{i \theta} S$ for suitably chosen $\theta$ to insure that this half-plane is $\operatorname{Re} z \geqq \epsilon$ for some $\epsilon>0$. Then, if $A=\frac{1}{2}\left(S+S^{*}\right)$, the numerical range of $A(=\operatorname{Re} W(S))$ lies on the real axis to the right of $\epsilon$, hence $A$ is positive and invertible. Since $T A=A T^{*}$, it follows that $A^{-1 / 2} T A^{1 / 2}$ is selfadjoint.

The proof of Theorem 2 shows that if $T S=S T^{*}$ where $S$ is positive and invertible, then $T$ is similar to a selfadjoint operator. Both assumptions on $S$ are essential here. Thus, Example 1 shows that the positivity condition on $S$ cannot be omitted, and Dieudonné [3] has given an example which shows that the conditions $T S=S T^{*}, S>0$ do not imply that the spectrum of $T$ is real.

The conclusion of Theorem 2 cannot be strengthened either:

Example 2. The conditions $S^{-1} T S=T^{*}, 0 \notin W(S)^{-}$do not imply that $T$ is normal.

Here it suffices to take $T=S B$ where $S$ is positive, $B$ is selfadjoint, and $S$ and $B$ do not commute.

The converse of Theorem 2 is also valid. That is, if $T$ is similar to a selfadjoint operator, then $T$ is similar to $T^{*}$ and the similarity can be implemented by an $S$ with $0 \notin W(S)^{-}$.

Proof. If $R^{-1} T R$ is selfadjoint, then $\left(R R^{*}\right)^{-1} T\left(R R^{*}\right)=T^{*}$, and $0 € W\left(R R^{*}\right)^{-}$because $R R^{*}$ is positive and invertible.

Thus Theorem 2 locates the scalar operators with real spectra in the larger class $\mathfrak{C}$ of operators which are similar to their adjoints.

In conclusion I would like to thank P. A. Fillmore for a stimulating 
discussion of Sheth's Theorem. He supplied Example 1. (The referee has observed that the same example occurs in the review of Sheth's paper [MR 33 \#4685].)

Note ADDED IN PROOF. A paper of C. A. McCarthy [J. London Math. Soc. 39 (1964), 288-290] contains another generalization of the theorem of Beck and Putnam.

\section{REFERENCES}

1. W. A. Beck and C. R. Putnam, A note on normal operators and their adjoints, J. London Math. Soc. 31 (1956), 213-216.

2. S. K. Berberian, $A$ note on operators unitarily equivalent to their adjoints, J. London Math. Soc. 37 (1962), 403-404.

3. J. Dieudonné, Quasi-hermitian operators, Proc. Internat. Sympos. Linear Spaces (Jerusalem, 1960), Jerusalem Academic Press, Jerusalem; Pergamon, Oxford, 1961, pp. 115-122.

4. I. H. Sheth, On hyponormal operators, Proc. Amer. Math. Soc. 17 (1966), 998-1001.

INDIANA UNIVERSITY 